\title{
Closed formulae to determine the angular velocity of a body-segment based on 3D measurements
}

\author{
L. Kocsis, G. Béda
}

Department of Applied Mechanics, Budapest University of Technology and Economics, Budapest, Hungary

Received: March 7, 2001

Accepted: May 14, 2001

This paper suggests a simple method to determine the global coordinates of the angular velocity and the angular acceleration of a body segment determined by the coordinates of minimum three markers. There are commonly used calculations for the angular quantities basing on the "hypothesis" of planar motion. The usage of approximate methods can result in quantitative and qualitative errors that may completely disort the reality. The method mentioned here is theoretically absolutely correct and can be well used for smoothing noisy data.

Keywords: kinematics, global coordinate system, angular quantities, angular acceleration

In experimental research the location and the orientation of the body-segments are determined from the spatial coordinates of markers attached to the body-segments. To determine the correct orientation, no less than three not collinear markers must be applied at one segment. There are methods to reduce the effect of the noise and to determine the derivatives of the measured global coordinates, but no theoretically correct and easily used method is known to determine the coordinates of the angular velocity and the angular acceleration of the segment, based directly on the measured global coordinates of the markers. Various coordinate systems are used to describe the body position as clinical system, globographic presentation, segment coordinate system and the joint rotation convention (7).

Correspondence should be addressed to

László Kocsis

Department of Applied Mechanics

Budapest University of Technology and Economics

H-1111 Budapest, Múegyetem rkp. 5, Hungary

Phone: (36-1) 463-1368

E-mail: kocsis@mm.bme.hu 
Improvements in computer technology have enabled us to rapidly analyze human movement patterns. There are a lot of professional software and hardware packages (2) that are able to determine the spatial coordinates of the required points within a given error rate. These systems are using active markers (ZEBRIS, Selspot) or passive ones (video-based systems) for determining the spatial coordinates of the key points of the body-segment. Most of the video-based systems (VICON, APAS, Mikromak, Peak, Simi Motion, Novel) are working with $50-60 \mathrm{~Hz}$, but there are also some systems that work with 100-1000 Hz. Using smoothing algorithms the velocities and the accelerations of the key points can be determined by the required accuracy. Nowadays only a few systems are able to determine the problem of inverse dynamics (determination of the forces and moments acting at the joints of the body-segments). To be able to solve this problem, the correct values of the angular velocity and the angular acceleration must be known. The users - reducing the errors of digitization - generally use only two markers at each segment.

In this case, there is no possibility to determine the correct values of the angular velocity and angular acceleration, except in case of real planar motion. Most of the professional packages determine the angular velocity and the angular acceleration of the segments by differentiation of the component angles obtained from the projections of the segment on the coordinate-planes. This method is not correct. This is well known for engineers and physicians as Budo already mentioned in his book in 1953 (3), but does not seem to be widely known in biomechanical applications. Many researchers use the change in position of the projection of a body segment onto a plane of interest (usually the film plane) to determine the component of angular velocity in the plane. M. Ramey and C. Nicodemus (4) wrote a note to illustrate that the determination of angular velocity components in this manner leads to erroneous values except in some special cases, but to date the simplification mentioned has been in use.

There are systems (5) where three markers are in use at each segment, and for the determination of the angular velocity and angular acceleration the Euler angles are used. This is a very complicated calculation and the values are determined in the local coordinate systems connected being to the segments and the whole model must be used. M. J. L. Alexander and J. Colbourne developed a method (1) to calculate the angular velocity of a body segment. Their formulae is really simple, and it works well in case of quick movements (they developed it for the calculation of a high-speed throwing motion), but near to zero value of the angular velocity the formulae cannot be used correctly, because in the denominator the value becomes to zero. M. C. Verstraete and R. W. Soutas-Little (6) developed an other correct method. Their method is based on the Method of Least Squares, and minimum 4 markers must be applied on a segment. In case of active marker's systems this will widely reduce the range of measurement, and for video-based systems the manual digitalization time will be increased. 
In this paper a new method will be demonstrated to determine the correct values of the angular velocity and angular acceleration of the segments using the velocities and the accelerations of the markers (key points) in the global coordinate system of the measuring unit. These data could be determined by any of the previously mentioned systems, using arbitrary positioned "triple set" investigating the body-segment. Most of the scientists like to post-process the measured data alone, according to the needs and not depending on the complete biomechanical model, provided by the used software package.

\section{Methods}

At the investigation of the human movement we generally suppose that the bodysegments are rigid bodies connecting to each other at the joints. The state of velocity of a rigid body is known if the velocity of any point and the angular velocity of the body are given. Let's suppose the point is the point $\mathrm{A}$, and we know its velocity $\underline{v}_{A}$ and the angular velocity $\underline{\omega}$.

To determine the state of acceleration the angular velocity $\underline{\omega}$, the angular acceleration $\underline{\varepsilon}$, and the acceleration of the point $\mathrm{A} \underline{a}_{A}$ must be known.

Investigating the state of velocity, let's suppose we know $\underline{v}_{A}, \underline{v}_{B}$ and $\underline{v}_{C}$, the velocities of $A, B, C$ points (5), which points are not collinear (Fig. 1).

The velocities of the points $\mathrm{B}$ and $\mathrm{C}$ can be expressed by $\underline{v}_{A}$ and $\underline{\omega}$.

$$
\begin{aligned}
& \underline{v}_{B}=\underline{v}_{A}+\underline{\omega} \times \underline{r}_{A B} \\
& \underline{v}_{C}=\underline{v}_{A}+\underline{\omega} \times \underline{r}_{A C}
\end{aligned}
$$

$\underline{r}_{A B}$ and $\underline{r}_{A C}$ are the position vectors pointing from $A$ to $B$ and from $A$ to $C$.

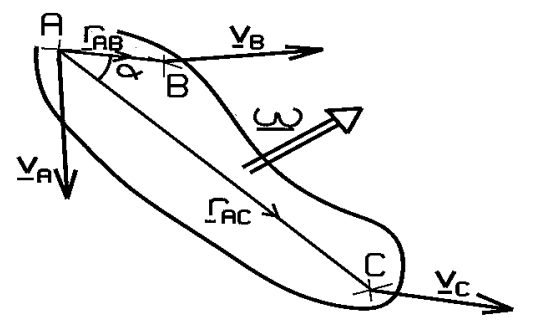

Fig. 1. The body-segment with the three markers and their velocities

The purpose of the following arrangements is to express $\underline{\omega}$. After rearranging [1] and [2], take the vector product from left by $\underline{r}_{A B}$ and by $\underline{r}_{A C}$, respectively: 


$$
\begin{aligned}
& \underline{r}_{A B} \times\left(\underline{v}_{B}-\underline{v}_{A}\right)=\underline{r}_{A B} \times\left(\underline{\omega} \times \underline{r}_{A B}\right) \\
& \underline{r}_{A C} \times\left(\underline{v}_{C}-\underline{v}_{A}\right)=\underline{r}_{A C} \times\left(\underline{\omega} \times \underline{r}_{A C}\right)
\end{aligned}
$$

By expressing the right sides of the equations we get:

$$
\begin{aligned}
& \underline{\underline{r}}_{A B} \times\left(\underline{v}_{B}-\underline{v}_{A}\right)=r^{2}{ }_{A B} \underline{\omega}-\left(\underline{r}_{A B} \cdot \underline{\omega}\right) \underline{r}_{A B} \\
& \underline{r}_{A C} \times\left(\underline{v}_{C}-\underline{v}_{A}\right)=r^{2}{ }_{A C} \underline{\omega}-\left(\underline{r}_{A C} \cdot \underline{\omega}\right) \underline{r}_{A C}
\end{aligned}
$$

Take the scalar product of [3] by $\underline{r}_{A C}$ and of [4] by $\underline{r}_{A B}$, after rearrangements:

$$
\left(\underline{r}_{A C} \underline{r}_{A B}\right) \cdot\left(\underline{v}_{B}-\underline{v}_{A}\right)=r_{A B}^{2}\left(\underline{\omega} \cdot \underline{r}_{A C}\right)-\left(\underline{r}_{A B} \cdot \underline{\omega}\right)\left(\underline{r}_{A C} \cdot \underline{r}_{A B}\right)
$$

and

$$
\left(\underline{r}_{A B} \times \underline{r}_{A C}\right) \cdot\left(\underline{v}_{C}-\underline{v}_{A}\right)=r_{A C}^{2}\left(\underline{\omega} \cdot \underline{r}_{A B}\right)-\left(\underline{r}_{A C} \cdot \underline{\omega}\right)\left(\underline{r}_{A C} \cdot \underline{r}_{A B}\right)
$$

In equations [5] and [6] the unknowns are $\underline{\omega} \underline{r}_{A C} \equiv \underline{r}_{A C} \cdot \underline{\omega}$ and $\underline{\omega} \cdot \underline{r}_{A B} \equiv \underline{r}_{A B} \cdot \underline{\omega}$. According to these unknowns the equations [5] and [6] are linear inhomogeneous algebraic equations. $D$ is the determinant of this system:

$$
D=\left|\begin{array}{cc}
r_{A B}^{2} & -\underline{r}_{A C} \cdot \underline{r}_{A B} \\
-\underline{r}_{A C} \cdot \underline{r}_{A B} & r_{A C}^{2}
\end{array}\right|=r_{A B}^{2} r_{A C}^{2}-\left(\underline{r}_{A C} \cdot \underline{r}_{A B}\right)^{2}
$$

or

$$
D=\overline{A B}^{2} \overline{A C}^{2} \sin ^{2} \alpha,
$$

where $\overline{A B} \equiv b$ and $\overline{A C} \equiv c$ are the distances between $A$ and $B$ and $A$ and $C$, respectively. $\alpha$ is the angle between the line segments $A B$ and $A C$.

Solving the system of equations [5] and [6] substituting - for instant $-\underline{\omega} \cdot \underline{r}_{A C}$ into the equation [4] we can get:

$$
\underline{\bar{\sigma}}=\frac{1}{c^{2}}\left\{\underline{r}_{A C} \times\left(\underline{v}_{C}-\underline{v}_{A}\right)+\left[\frac{\underline{r}_{A B \times} \times \underline{r}_{A C}}{b \sin ^{2} \alpha} \cdot\left(\frac{\underline{v}_{C}-\underline{v}_{A}}{c} \cos \alpha-\frac{\underline{v}_{B}-\underline{v}_{A}}{b}\right)\right] \underline{r}_{A C}\right\} .
$$

The state of acceleration of a rigid body is determined if we know $\underline{\omega}$, and the accelerations of three non-collinear points. The angular acceleration $\underline{\varepsilon}$ can be determined by differentiating the equation [7]:

$$
\begin{gathered}
\underline{\varepsilon}=\frac{1}{c^{2}}\left\{\underline{r}_{A C} \times\left[\underline{a}_{C}-\underline{a}_{A}-\left(\underline{\omega}-\underline{r}_{A C}\right) \underline{\omega}\right]+\frac{\underline{r}_{A B \times} \times \underline{r}_{A C} .}{b \sin ^{2} \alpha}\right. \\
\left.\cdot\left[\frac{\underline{a}_{C}-\underline{a}_{A}-\left(\underline{\omega} \cdot \underline{r}_{A C}\right) \underline{\omega}}{c} \cos \alpha-\frac{\underline{a}_{B}-\underline{a}_{A}-\left(\underline{\omega} \cdot \underline{r}_{A B}\right) \underline{\omega}}{b}\right] \underline{r}_{A C}\right\} .
\end{gathered}
$$




\section{Results and Discussion}

Spastic hemiparesis mostly arises as a consequence of stroke, brain injury, multiple sclerosis, brain tumor, perinatal damage. In these cases not only the muscular strength becomes weaker, but the muscular tone increases causing further problems.

REHAROB project (IST-1999-13109 REHAROB = Supporting Rehabilitation of Disabled Using Industrial Robots for Upper Limb Motion Therapy) will be a robotic rehabilitation system for upper limb motion therapy for the disabled. Industrial robots, utilizing intelligent identification of the required physiotherapy motion will drive the therapy. The REHAROB project will provide personalized three-dimensional motion therapy for patients with neuro-motor impairments. The REHAROB will provide an online physiotherapy monitoring and documentation system with 3D motion therapy measuring, visualizing and logging. Exercises were collected by physiotherapists working for the REHAROB project and than described in a catalogue. The robot will be required to execute selected exercises from this catalogue. The aim of these exercises is to improve the patient's upper limb movements (strengthen the muscles, decrease spasticity, improve coordination and proprioception, increase the range of movement of the joints).

We specially developed the mentioned method to characterize these exercises, but the formulae can be widely used in general motion analysis. Let's choose from these exercises a simple one, as an example (Fig. 2).

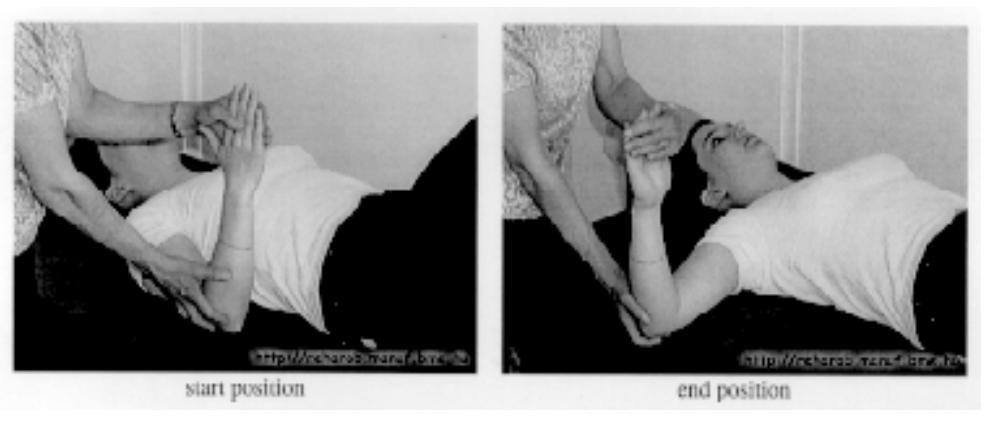

Fig. 2. Start and end position

Starting position: The patient lies on his back.

Upper arm lies on the bed next to the body, elbow is in a 90degree flexion, palm is turned towards the body (0-degree position). 
Line of movement: Continuous upper arm abduction between the starting and the end position.

End position: $\quad$ 90-degree position of upper arm.

Execution of movement: Slow, continuous movement.

The calculation based on a measurement of the described exercise, using 3 markers at the upper arm according to Figure 3. On the basis of the measurement we have the positions, velocities and accelerations versus time functions as series of data, getting from the Zebris system CMS70P. Based on these data we determine the angular velocity and angular acceleration versus time functions as mentioned in this paper.

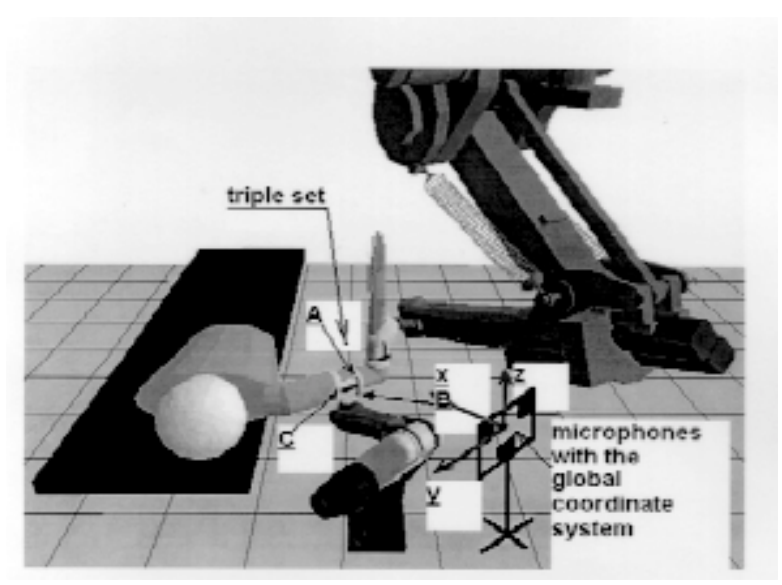

Fig. 3. Arrangement of the markers and the microphones at the measurement using the ZEBRIS CMS-HS measuring system

Figure 4 shows the three components of the angular velocity for the previously described exercise in the global coordinate system of measuring unit, as demonstrated in Figure 3.

According to the measured exercise the microphones and the marker arrangements are always different, and the measured data will be transformed from the global coordinate system of the measuring unit to the special coordinate systems of the used robots (Fig. 3). 


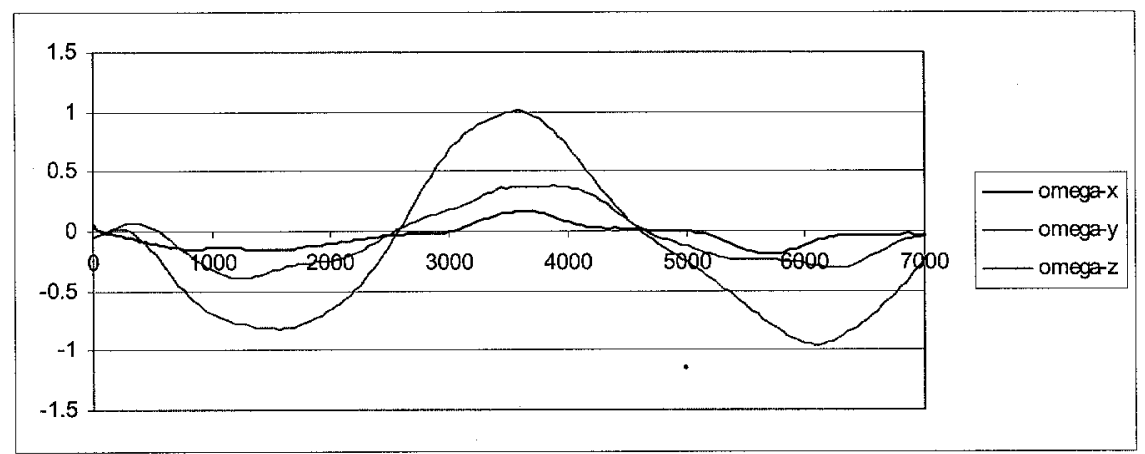

Fig. 4. The three components of the angular velocity $[1 / \mathrm{s}]$ versus time [msec] functiones for the exercise shown in Figure 2

\section{Example 1 (Theoretical control of the formulae)}

Let's put a cone with a length $1=1 \mathrm{~m}$, and with a radius of its base circle $R=0.5 \mathrm{~m}$ into a Descartesien coordinate system according to the arrangements of Figure 5. The apex coincides with the origin and the cone's axis of rotation lies in the $\mathrm{y}-\mathrm{z}$ plane. Let's rotate first the cone about its axis of rotation with constant angular velocity $\omega_{f}=4 / s$, than rotate the cone about the $\mathrm{x}$ axis with the angular velocity $\omega_{x}=2+5 t$. (The axis of the cone moves in the $\mathrm{y}-\mathrm{z}$ plane!)

Let's determine the angular velocity and angular acceleration versus time functions of the cone on the basis of the position, velocity and acceleration of three points $(A, B, C)$ lying on the base circle of the cone.

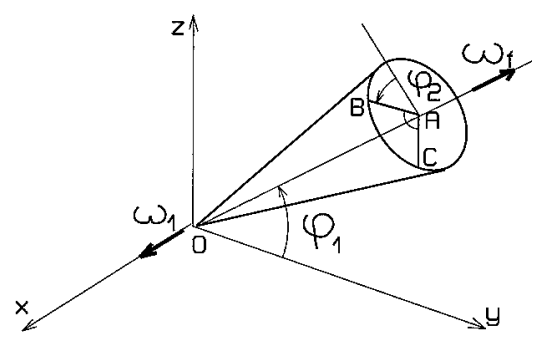

Fig. 5. Demonstration of the cone for Exercise 1 
In this case the scalar coordinates of the angular velocity can be determined theoretically:

$\omega_{x}=2+5 t$

$\omega_{y}=4 \cos \phi_{1}$

$\omega_{z}=4 \sin \phi_{1}$

Using the equation [7], the results are exactly the same as can be seen in Figure 5 (Fig. 6).

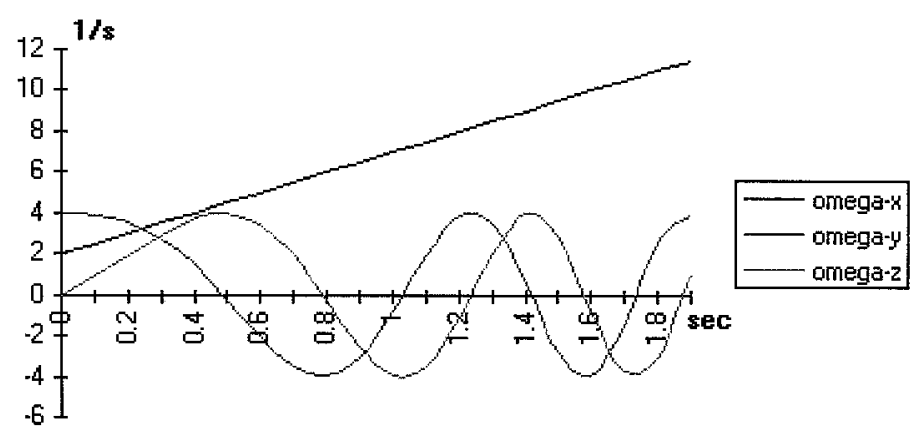

Fig. 6. Angular velocity-time functions of the cone determined by equation [7]

\section{Example 2}

The following calculation based on a measurement of the thigh of a walking woman on a treadmill with a speed of $2 \mathrm{~km} / \mathrm{h}$, used 3 markers $(4=r$. tibial tubercle, $6=\mathrm{r}$. femoral epicondyle, $8=\mathrm{r}$. greater trochanter) determining the spatial position of the body-segment). On the basis of the measurement we have the positions versus time functions as series of data (Fig. 7). Based on this data we determined the angular velocity and angular acceleration versus time functions of the thigh (Figs 8-10).

Figures 8-10 compare the results of the "correct" calculations described in this paper, according to the "planar" ones during the investigated interval of the gait, to get an idea about the errors caused by the simplification.

Investigating these Figures one can recognize if the motion is very near to a planar motion, the errors between the two calculations can be neglected (see Fig. 9), and this is the purpose why the "planar" method is still in use. In case of the other coordinates not only the values are different but the characters of the functions are also different. According to these diagrams one can draw false inference. 


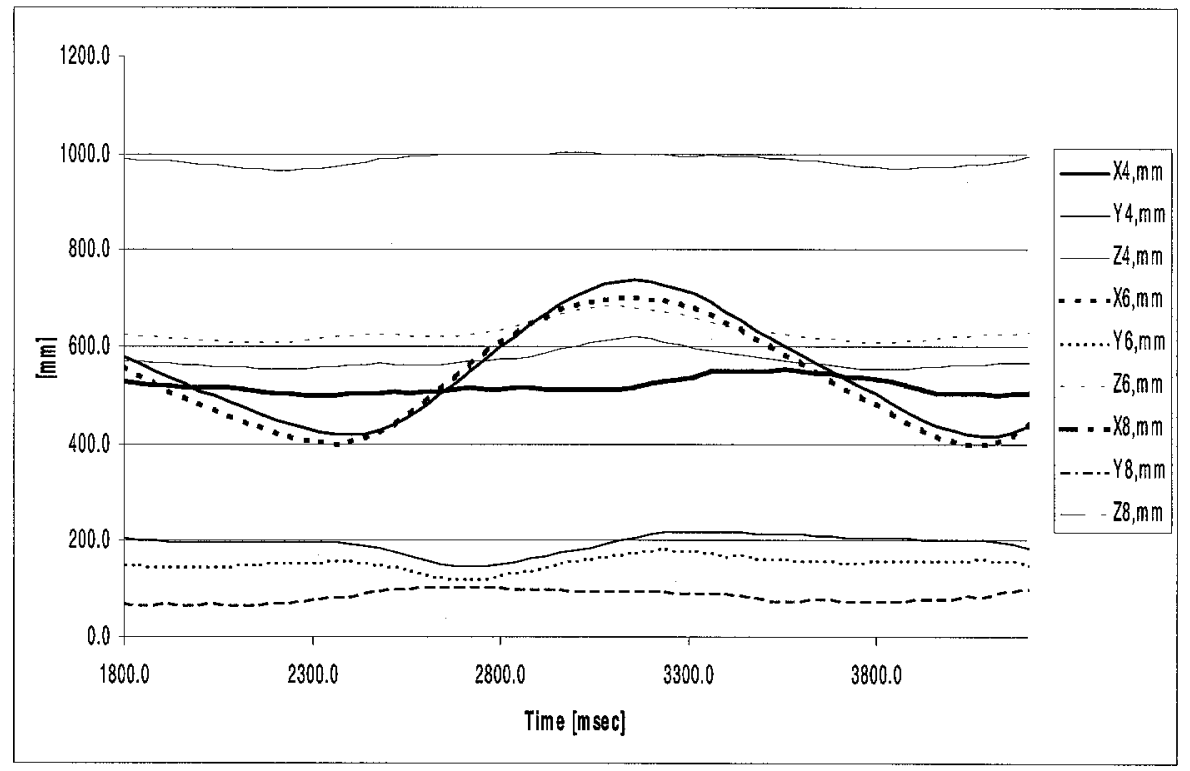

Fig. 7. Coordinates - versus time functions of the markers during gait

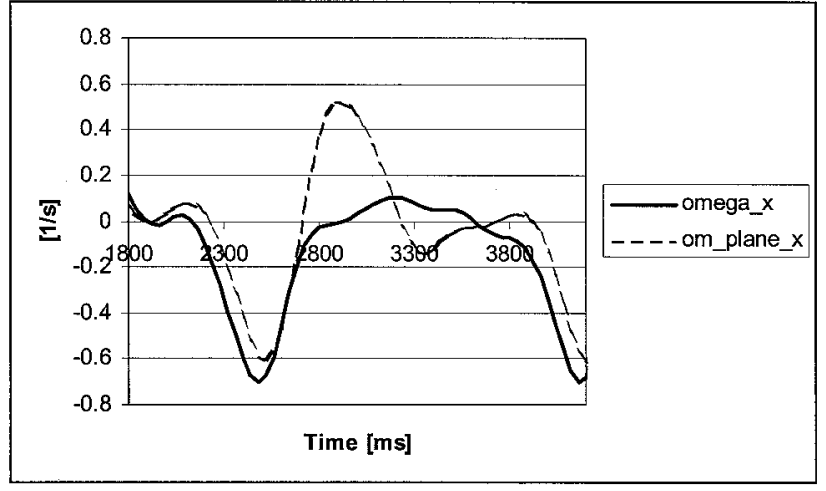

Fig. 8. The changes of the $\mathrm{x}$ coordinate of the angular velocity (omega_ $\mathrm{x}=$ the correct calculation, om_plane_x $=$ the value based on the component motion $\mathrm{x}$ axis shows towards the direction of the motion) 


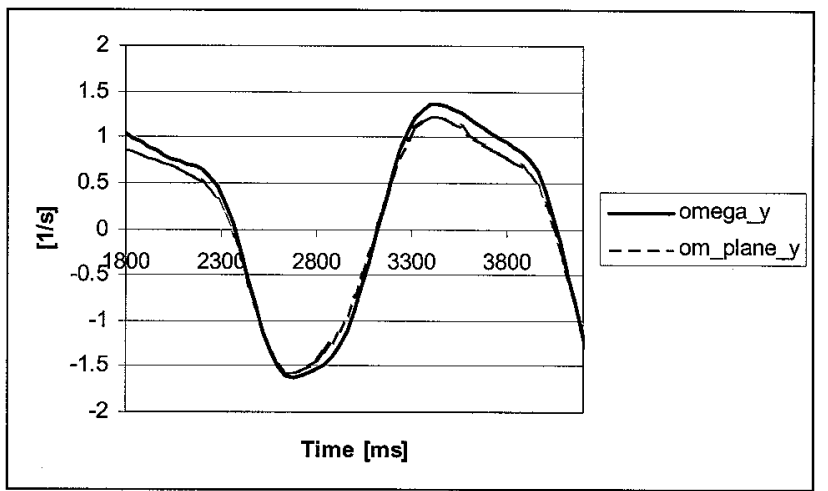

Fig. 9. The changes of the $y$ coordinate of the angular velocity (omega_y $=$ the correct calculation, om_plane_y $=$ the value based on the component motion y axis is horizontal, perpenducular to the direction of the motion)

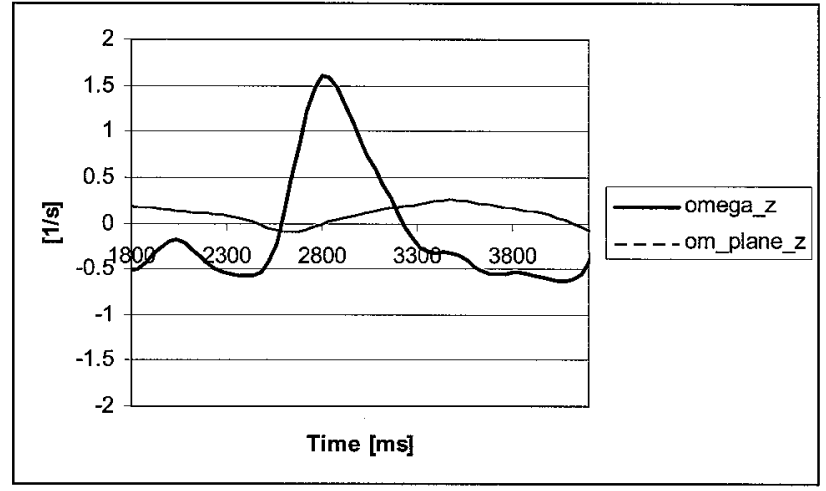

Fig. 10. The changes of the $\mathrm{z}$ coordinate of the angular velocity (omega_ $\mathrm{z}=$ the correct calculation, om_plane $\_\mathrm{z}=$ the value based on the component motion $\mathrm{z}$ axis is vertical)

\section{Conclusion}

Advantage of the suggested method is the simplicity of the calculations comparing with the other methods mentioned $(1,6)$. This can be recognized seeing the scalar equations in the Appendix. There is no need to use very complicated 
transformations, which will cause extra errors in the data, and all the calculations are based on the global coordinates of the markers. Using systematical permutation of A, B and $\mathrm{C}$, six different calculations could be made on the same data basis of three markers. In case of "smooth" data these results are identical. Applying these calculations for noisy data, the results will alternate a bit, but the average of these functions will insure a smoother solution, as we would use instead of three markers on the same body, as the Method of Least Squares (Fig. 11). demonstrates the mentioned six different solutions and their average for the $\mathrm{x}$ component of the angular velocity. The results, according to the needs of the users can be further smoothed and transformed. The authors hope that this method will be used widely to determine the correct values of the coordinates of the angular velocity for a body-segment.

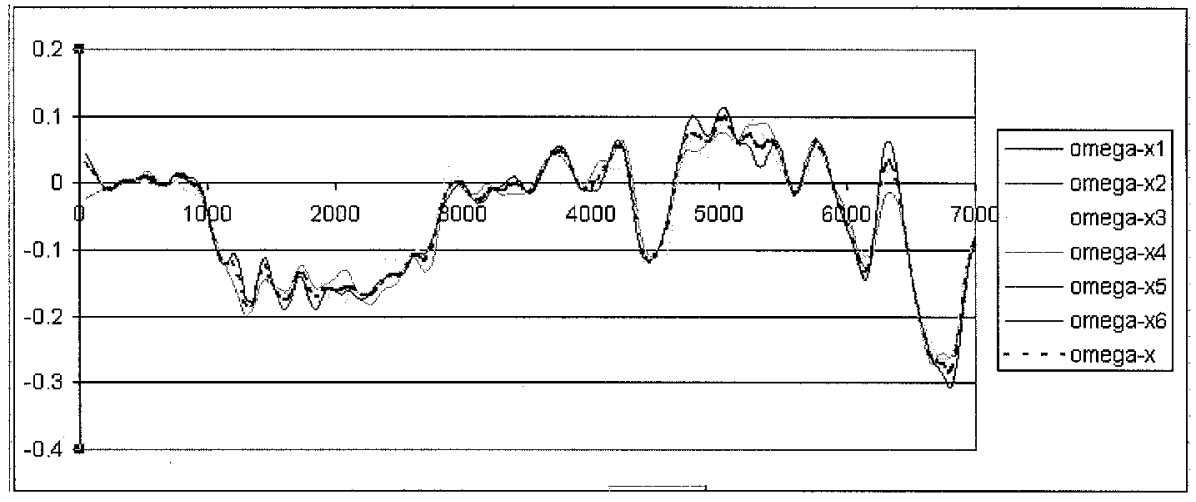

Fig. 11. "noisy" $\mathrm{x}$ components of the angular velocity $[1 / \mathrm{s}]$ versus time $[\mathrm{msec}]$ funcions in case of systematic permutation of equation [7] and their average (omega-x)

\section{Appendix}

\section{Scalar equations to determine the angular velocity}

Since all, in equation [7] mentioned coordinates and velocities are determined in the global $x, y, z$ coordinate system, the coordinates of $\underline{\omega}$ will be determined in the same coordinate system. Let's denote the coordinates of the point A by $x_{A}, y_{A}$ and $z_{A}$, the coordinates of the velocity of the same point by $v_{A x}, v_{A y}, v_{A z}$, and using similar 
notations in case of the points B and C. Finally the coordinates of $\underline{\omega}$ are denoted by $\omega_{x}$, $\omega_{y}, \omega_{z}$, respectively. The coordinates of $\underline{\omega}$ are:

$$
\begin{aligned}
& \omega_{x}=\frac{1}{c^{2}}\left[n_{1}+\frac{D_{1}}{D}\left(x_{C}-x_{A}\right)\right], \\
& \omega_{y}=\frac{1}{c^{2}}\left[n_{2}+\frac{D_{1}}{D}\left(y_{C}-y_{A}\right)\right], \\
& \omega_{z}=\frac{1}{c^{2}}\left[n_{3}+\frac{D_{1}}{D}\left(z_{C}-z_{A}\right)\right],
\end{aligned}
$$

here:

$$
\begin{aligned}
& n_{1}=\left(y_{C}-y_{A}\right)\left(v_{C z}-v_{A z}\right)-\left(z_{C}-z_{A}\right)\left(v_{C y}-v_{A y}\right), \\
& n_{2}=\left(z_{C}-z_{A}\right)\left(v_{C x}-v_{A x}\right)-\left(x_{C}-x_{A}\right)\left(v_{C z}-v_{A z}\right) \\
& n_{3}=\left(x_{C}-x_{A}\right)\left(v_{C y}-v_{A y}\right)-\left(y_{C}-y_{A}\right)\left(v_{C x}-v_{A x}\right)
\end{aligned}
$$

and

$$
\begin{aligned}
& D_{1}=c^{2}\left[m_{1}\left(v_{B x}-v_{A x}\right)+m_{2}\left(v_{B y}-v_{A y}\right)+m_{3}\left(v_{B z}-v_{A z}\right)\right]- \\
& -b c \cos \alpha\left[m_{1}\left(v_{C x}-v_{A x}\right)+m_{2}\left(v_{C y}-v_{A y}\right)+m_{3}\left(v_{C z}-v_{A z}\right)\right]
\end{aligned}
$$

where:

$$
\begin{gathered}
m_{1}=\left(y_{C}-y_{A}\right)\left(z_{B}-z_{A}\right)-\left(y_{B}-Y_{A}\right)\left(z_{C}-z_{A}\right) \\
m_{2}=\left(x_{b}-x_{A}\right)\left(z_{C}-z_{A}\right)-\left(x_{C}-x_{A}\right)\left(z_{B}-z_{A}\right) \\
m_{3}=\left(x_{C}-x_{A}\right)\left(y_{B}-y_{A}\right)-\left(x_{b}-x_{A}\right)\left(y_{C}-y_{A}\right), \\
D=b^{2} c^{2} \sin ^{2} \alpha=b^{2} c^{2}\left(1-\cos ^{2} \alpha\right) \\
b^{2}=\left(x_{B}-x_{A}\right)^{2}+\left(y_{B}-y_{A}\right)^{2}+\left(z_{B}-z_{A}\right)^{2}, \\
c^{2}=\left(x_{C}-x_{A}\right)^{2}+\left(y_{C}-y_{A}\right)^{2}+\left(z_{C}-z_{A}\right)^{2} \\
\cos \alpha=\frac{\left(x_{B}-x_{A}\right)\left(x_{C}-x_{A}\right)+\left(y_{B}-y_{A}\right)\left(y_{C}-y_{A}\right)+\left(z_{B}-z_{A}\right)\left(z_{C}-z_{A}\right)}{b \cdot c}
\end{gathered}
$$




\section{REFERENCES}

1. Alexander, M. J. L., Colbourne, J. (1980): A method of determination of the angular velocity vector of a limb segment JAB.

2. Allard, P., Cappozzo, A., Lundberg, A., Vaughan, Ch. L. (1997): Three-dimensional Analysis of Human Locomotion. Wiley.

3. Budó, A. (1953): Mechanika. Tankönyvkiadó, Budapest.

4. Ramey, M., Nicodemus, C.: A note on the determination of angular velocities in human motion studies. Medicine and Science in Sports 9 (2), 134-136 (1977).

5. Vaughan, Ch. L., Davis, B. L., O’Connor, J. C.: Dynamics of Human Gait. Human Kinetics (1992).

6. Verstraete, M. C., Soutas-Little, R. W.: A method for computing the three-dimensional angular velocity and acceleration of a body-segment from three-dimensional position data. Journal of Biomechanical Engineering 112, 114-118 (1990).

7. Zatsiorsky, V. M. (1998): Kinematics of Human Motion. Human Kinetics. 